\title{
PAVEMENT CONDITION AND TRAFFIC INDICES FOR PRIORITIZING ROAD MAINTENANCE
}

\author{
Georgios M. Hadjidemetriou ${ }^{1}$, Michalis Tsangaris ${ }^{2}$, and Symeon Christodoulou ${ }^{2}$ \\ ${ }^{1}$ University of Cambridge, Cambridge, United Kingdom \\ ${ }^{2}$ University of Cyprus, Nicosia, Cyprus
}

\begin{abstract}
Maintenance prioritization is a regular challenge that transportation departments face. They periodically have to choose among many roadway sections the ones that take precedence over others when it comes to the order of rehabilitation. They typically follow methodologies that consider only pavement condition for prioritizing roadways, without considering the number of the users. Presented herein is the way information provided by automated techniques for pavement and traffic evaluation is transformed into a numerical metric that can be utilized for maintenance prioritization purposes. The contribution of the proposed system can be summarized in: (1) the implementation of two entropy-based classification approaches (change-point detection and thresholding) for detecting "distressed pavement" areas; (2) the development of a pavement condition index that is a continuous variable; and (3) the creation of maintenance prioritization index based on both pavement condition and traffic.
\end{abstract}

\section{Introduction}

Maintenance prioritization of roadway sections is essential, especially in case of budget constraints. Departments of Transportation (DOTs) select amongst several road sections the ones that take priority over others when it comes to the order of maintenance. Most DOTs follow procedures that take into account only pavement condition (Bandara and Gunaratne, 2001; Pantha et al., 2010) for prioritizing roadways of the same type (e.g. highways). They utilize the International Roughness Index (IRI) measurement, a pavement condition index, or a combination of the two.

The former is the most commonly used index for measuring road roughness, defined as the divergence of a roadway surface from a planar surface, affecting vehicle dynamics, drainage ride quality, and dynamic loads (ASTM International, 2012). The latter is normally calculated based on the type, severity and extent of specific defects, existing in the examined section. PASER and PSCI are two examples of pavement condition indices. The former consists the condition index for Michigan DOT (Walker, 2002), while the latter is used by the (Department of Transport, Tourism and Sport, Government of Ireland, 2013). PACER and PSCI are numerical indices between 1 and 10 , with 10 indicating excellent condition and 1 showing failure.

However, in recent years several researchers have reconsidered the maintenance processes, claiming that a priority-of-work analysis should be a multi-criteria procedure that determines the optimal ranking list of candidate segments for maintenance based on numerous factors (Shah et al., 2014).

Ndume and Mlavi (2017) claimed that the most important factors affecting road maintenance prioritization should be: traffic, population served, production centers, social services, connectivity, road class and tourism. Though, this study does not consider pavement condition, ignoring that if a road section is economically important but in an excellent condition, it should not be prioritized over a section that is less economically important but its condition threatens passengers' safety.

Reddy and Veeraragavan (2001) developed a maintenance priority index concept that is based on an overall distress index model and traffic adjustment factors. In addition, Moazami et al. (2011) claimed that the factors needed to be included in a prioritization model consist of pavement condition index, traffic volume, maintenance and rehabilitation cost, as well as road width. Another study named the following elements to be considered by a priority ranking model: pavement serviceability and condition index, traffic level, functional classification, and agency cost (Fwa, 2005). However, these studies do not include all information needed on the procedures of collecting and processing data to estimate these variables.

Pavement condition and traffic volume, which comprise the common factors in the aforementioned studies, are those that require the most time and cost to be estimated. Several researchers have attempted to contribute on automated pavement condition and traffic assessment since current manual techniques are time-consuming, subjective and pose safety risks for surveyors (Bianchini et al., 2010).

Pavement evaluation methods in literature can be divided based on the way data is collected. Data can be collected via vibration-based sensors that are embedded in smartphones and vehicles, or via cameras and laser scanners positioned on moving vehicles, followed by data processing using computer-vision techniques. Lakušić et al. (2011), Yu and Yu (2006), 
Ngwangwa et al., (2014) and Harris et al. (2010) utilized accelerometers to estimate road roughness or characterize road profile, without classifying specific defect types. Mednis et al. (2011), Douangphachanh and Oneyama (2014), and Kumar et al. (2017) utilized accelerometers embedded on android smartphones. The former study detects potholes, while the other two relate road roughness condition with acceleration vibration. The exploitation of gyroscopes, combined with other sensors embedded on smartphones, was investigated by Seraj et al. (2014) and Mohamed et al. (2015), detecting severe pavement defects. Vibrationbased methods have been proved effective in estimating road roughness and detecting "elevation" defects, such as potholes. However, they miss pavement defects if the moving vehicle, equipped with the sensors, do not pass over the defects, they are unable to detect defects which are not related to elevation differences (e.g. bleeding) and to classify the severity of defects that is based on visual characteristics (e.g. width of cracks).

A noteworthy number of researchers have concentrated on vision-based methodologies to avoid the aforementioned limitations. Zhou et al. (2006), Lokeshwor et al., (2014) Doycheva et al. (2016), (Yan and Yuan (2017), and Banharnsakun (2017) developed algorithms that detect distressed pavement areas, without classifying defect types. A significant number of other studies have concentrated on directly classifying specific defect types, such as cracking (Amarasiri et al., 2010; Huang and $\mathrm{Xu}, 2006$; Ying and Salari, 2010), potholes (Koch et al., 2012; Ryu et al., 2015), patching (Hadjidemetriou et al., 2018; Hadjidemetriou et al., 2016) and raveling (Mathavan et al., 2014). A few studies have attempted to classify multiple pavement distress types simultaneously (Radopoulou and Brilakis, 2016; Xu et al., 2016). Li and Wang (2016) used an emerging 3D pavement data collection system to detect cracking with a multiseed fusion algorithm, while Zhang et al. (2018, 2017) developed the CrackNet, a deep-learning based software, for detecting pavement cracks on 3D asphalt pavement surfaces. Nonetheless, the aforementioned studies do not describe the way this information can be used for rating pavement condition and prioritizing road maintenance.

Numerous other studies concern with automated traffic monitoring. One of the most widespread practices in automated traffic monitoring consists of sensors embedded in the pavement, with the majority being inductive loop detectors. Nevertheless, this practice suffers from high installation and maintenance costs (Zhang et al. 2011). Chen et al., (2001) as well as Gupte et al., (2002) designed visionbased systems that automatically detect and track vehicles.

The current paper describes work in progress on the development of procedures for rating asphalt pavement condition of a road section using visionbased identification of distressed areas, and for grading traffic using vision-based detection and classification of vehicles. Lastly, it aims to combine the two grades providing a final rating, which can be used for prioritizing maintenance of roadway sections.

\section{Methodology \\ Entropy-based Pavement Condition Index}

A vision-based method for distressed pavement areas detection (Hadjidemetriou and Christodoulou, 2019), provides the data needed for the development of the novel pavement condition index described below. The input of this method consists of collected pavement surface videos from which frames are extracted, along with their geospatial location. Shadows are removed from the grayscale images using a method developed by Gong and Cosker (2016), which upon testing on images of pavements with shadows showed almost perfect performance in removing such shadows. The shadows were eliminated in 519 frames (95.8\%), partially removed in 13 frames $(2.4 \%)$, and remained in 10 frames $(1.8 \%)$ after processing 542 frames extracted from the collected videos for the presented distressed-areas detection algorithm.

The subsequent phase of the developed system consists of the calculation of entropy, in the context of image-processing, as firstly introduced by Shannon (1948).

$$
E \equiv \operatorname{entropy}(I)=-\sum_{1}^{N} * \log _{2}(p)
$$

The parameter $p$ in Equation 1 stands for the probability of occurrence and $N$ represents the number of classes in the analysis. In the current case of a grayscale image, $p$ is the number of pixels per color on a scale of 0 to 255 divided by the total pixel count of the frame, while $N$ is the number of color values in the analysis (256). Entropy reaches maximality at the equiprobability state (i.e. when all classes have equal probability of occurrence) and minimality when all events are assigned to a single class (Christodoulou et al., 2009). Given a grayscale image, entropy maximality corresponds to an equal number of pixels in each gray shade, and minimality corresponds to all pixels having the same grayscale shade (for instance a completely black image). Thus, the worse the pavement condition is, the higher the entropy value gets. This is a result of the smooth texture of healthy pavement (which includes similar shades of gray and consequently a low entropy value) and the rough texture of distressed pavement (which contains a higher number of gray shades and therefore a greater entropy value).

Video frames of healthy and distressed pavement were 
analyzed to automatically separate the two classes. Thus, the solution space and the complexity of the problem will potentially be reduced since transportation authorities will have to deal only with the images including anomalies. The "distressed pavement" frames detection was initially performed by use of the relative unconstrained least-squares importance fitting (RuLSIF) method (Liu et al. 2013; Yamada et al. 2013). RuLSIF is a statistical changepoint detection algorithm based on non-parametric divergence estimation between signal samples from two retrospective segments. The algorithm uses the relative Pearson divergence as a divergence measure and a method of direct density-ratio estimation.

Figure 1 and Figure 2 show two examples of the video signals analyzed, with the corresponding anomaly detection results from the RuLSIF method. Video signal 1 was known to have transverse cracking and raveling in frames 1215-1269 and 1578-1622, respectively, while video signal 2 included patching, cracking (longitudinal and transverse) and raveling in frames 343-367, 531-574, and 656-670, respectively.
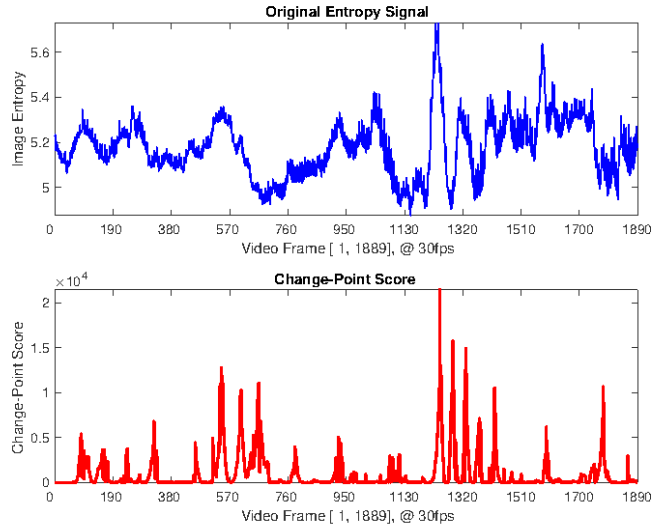

Figure 1: Entropy signal and change-point score of sample video 1
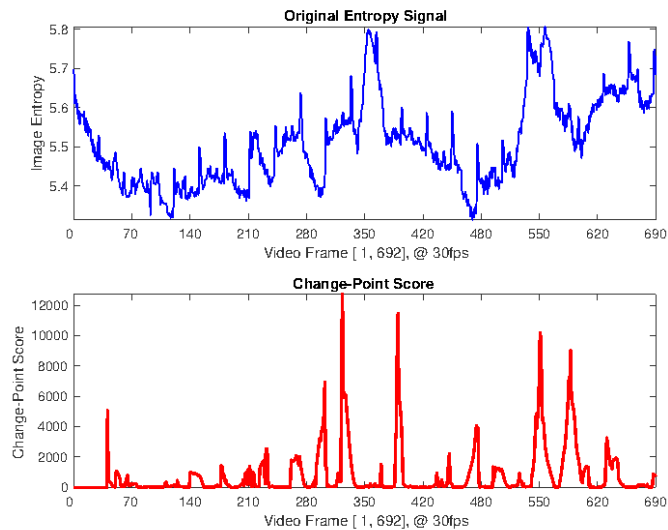

Figure 2: Entropy signal and change point score of sample video 2
The RuLSIF method identifies frames that have a significant change compared to the previous frames (regions where the change-point anomaly scores peak). In the current example, the algorithm proved accurate in identifying frames including anomalies (True Positives). However, in several cases it indicated healthy pavement areas as distressed areas (False Positives), when moving from a high number of consecutive "distressed pavement" frames to "healthy pavement" frames. False Positives increase the number of frames that authorities have to deal with, without achieving the initial aim of reduced time and complexity. Further, RuLSIF, as most change-point detection methods, even though better than traditional outlier detection methods (such as the moving average), fail when the transition between states is smooth and thus the pace of change (from one image to another) is small. In this case, there are "distressed pavement" frames identified as "healthy pavement" pavement frames (False Negatives). To avoid wrong indications and to increase the detection accuracy, the change-point detection approach was supplemented with a thresholding approach, as described below.

The final approach classifies frames into two classes, named "healthy pavement" and "distressed pavement", based on their entropy values. Frames that exceed a predefined entropy threshold value are classified as "distressed pavement". Figure 3 illustrates the entropy signal of a sample video with Xaxis and $\mathrm{Y}$-axis presenting the frame serial number and the frame entropy value respectively. The entropythresholding process extracts the "distressed pavement" frames, along with their geospatial location, with a classification accuracy of $89.2 \%$ (Hadjidemetriou and Christodoulou, 2019).

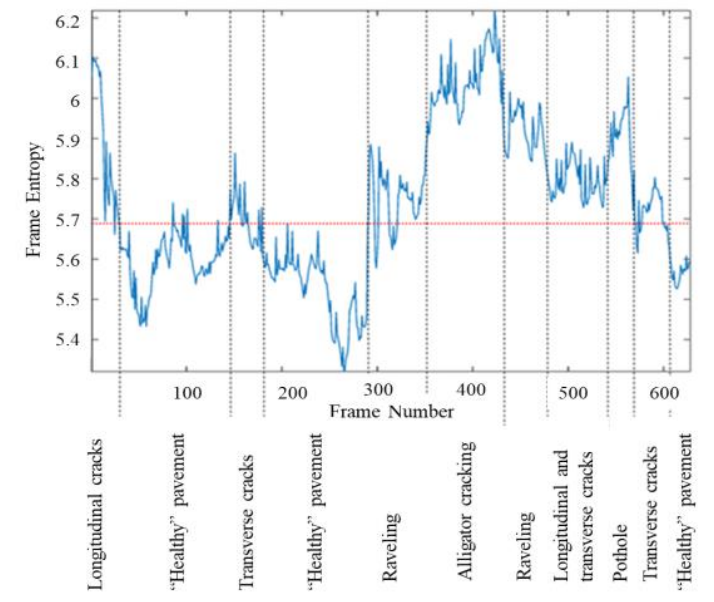

Figure 3: Entropy signal of the sample video, with annotations on the observed roadway defects (Hadjidemetriou and Christodoulou, 2019)

The aforementioned detection process provides the data needed for the development of a novel pavement 
Table 1: Comparison between PASER, PSCI and EPCI

\begin{tabular}{|c|c|c|c|c|c|}
\hline Section & Description of condition & PASER & PSCI & $\begin{array}{l}\text { Average } \\
\text { frame- } \\
\text { entropy }\end{array}$ & EPCI \\
\hline A & New construction & 10 & 10 & 5.42 & 1.00 \\
\hline B & Recent overlay, like new & 9 & 10 & 5.45 & 4.54 \\
\hline C & Low-severity raveling & 7 & 8 & 5.69 & 32.82 \\
\hline D & Medium-severity longitudinal cracking & 6 & 6 & 5.72 & 36.36 \\
\hline E & High-severity transverse cracking, low-severity & 5 & 5 & 5.76 & 41.07 \\
\hline F & Longitudinal cracking & & 5 & 5.8 & 45.79 \\
\hline G & High-severity raveling, medium-severity patching & 4 & 5 & 5.98 & 67.00 \\
\hline H & High-severity patches, high severity longitudinal and & 3 & 3 & 6.08 & 78.79 \\
\hline I & transverse cracking, high-severity raveling & & & & 88.21 \\
\hline J & Hoad disintegration of surface. Pavement failure & 1 & 1 & 6.26 & 100.00 \\
\hline
\end{tabular}

condition index, as presented below. The entropy values are normalized to a 1-100 scale and used as a condition rating index, while also calibrated against two of the most widespread pavement condition indices, called Pavement Surface Evaluation and Rating (PACER) and Pavement Surface Condition Index (PSCI). The pavement condition indices of PACER and PSCI were selected because: (a) they are currently used by transportation authorities; (b) the way they are used as well as their description for each level of the scale are clear; (c) they consider almost all possible pavement defects of asphalt pavements. The developed index is also divided into severity levels, with each level including possible pavement defect types.

The proposed system was tested by use of videos collected from roads (of approximate total length of 10 $\mathrm{km}$ ), separated into lane sections of $50 \mathrm{~m}$. The selection of the $50 \mathrm{~m}$-lane sections was based on a review of 56 different pavement manuals used by different DOTs, and on interviews of pavement engineers/experts. The entropy value of each frame is calculated using Equation 1, followed by the calculation of the average of entropy values for all images of the 50m-section. The average frame-entropy is then normalized to a scale from 1 to 100 (Equation 2 ) and compared with the PASER and PSCI indices. Figure 4 shows 10 representative frames collected from 10 different sections. Table 1 presents the defects that exist in these sections, the pavement condition rating by PASER and PSCI, the average frame entropy and its normalized value.

$$
x^{\prime}=a+\frac{(x-A)(b-a)}{(B-A)}
$$

x': normalized value

$\mathrm{x}$ : initial value

A: lowest entropy value in the dataset (i.e. 5.42)

B: highest entropy value in the dataset (i.e. 6.26)

a: lowest normalized value (1)

b: highest normalized value (100)

The comparison in Table 1 clearly shows that as the values of PASER and PSCI decrease, the normalized entropy value increases. Thus, the latter can be used as a pavement condition index, with similar results with the other two indices. It also has the advantage of being a continuous variable, compared to the other two indices that are integer and have only ten possible values. After reviewing all collected data, Table 2 was created to divide the entropy-based pavement condition index (EPCI) into levels. Each level characterizes the general pavement condition, while Table 2 also includes the possible pavement defects that might exist in every level, along with their severity level (low (L); medium (M); high (H)). The values of EPCI corresponding to possible defects were defined based on the conducted case study ( $10 \mathrm{~km}$-length road network). It should be noted that the current paper presents work in progress. Thus, these values need validation, testing the developed system in other road sections.

where: 
Table 2: Entropy- based pavement condition index

\begin{tabular}{|c|c|c|}
\hline $\begin{array}{l}\text { Entropy- } \\
\text { based } \\
\text { index }\end{array}$ & Pavement defects & $\begin{array}{l}\text { General } \\
\text { pavement } \\
\text { condition }\end{array}$ \\
\hline $1-32$ & No defects. & Healthy \\
\hline $33-35$ & Raveling or bleeding (L). Longitudinal or transverse cracking (L). & Very good \\
\hline $36-40$ & $\begin{array}{c}\text { Raveling or bleeding (M). Longitudinal or transverse cracking (M). Block } \\
\text { cracking (L) }\end{array}$ & Good \\
\hline $41-55$ & $\begin{array}{c}\text { Raveling or bleeding }(\mathrm{M}) \text {. Longitudinal or transverse cracking }(\mathrm{H}) . \text { Block } \\
\text { cracking }(\mathrm{M}) \text {. Edge cracking }(\mathrm{L}) \text {. Patching }(\mathrm{L})\end{array}$ & Fair \\
\hline $56-70$ & $\begin{array}{l}\text { Raveling or bleeding }(\mathrm{H}) \text {. Longitudinal or transverse or block cracking }(\mathrm{H}) \text {. Edge } \\
\text { cracking }(\mathrm{M}) \text {. Patching }(\mathrm{M}) \text {. Shoving or rutting or distortion }(\mathrm{L}) \text {. }\end{array}$ & Fair \\
\hline $71-80$ & $\begin{array}{l}\text { Raveling or bleeding }(\mathrm{H}) \text {. Longitudinal or transverse or block or edge cracking } \\
(\mathrm{H}) \text {. Alligator cracking }(\mathrm{L}) \text {. Patching }(\mathrm{H}) \text {. Shoving or rutting or distortion }(\mathrm{M}) \text {. } \\
\text { Potholes (L) }\end{array}$ & Poor \\
\hline $81-95$ & $\begin{array}{c}\text { Alligator cracking }(\mathrm{M} \text { or } \mathrm{H}) . \\
\text { Patching }(\mathrm{H}) . \text { Shoving or rutting or distortion }(\mathrm{H}) \text {. } \\
\text { Potholes }(\mathrm{M} \text { or } \mathrm{H})\end{array}$ & Very poor \\
\hline $95-100$ & Disintegration of surface & Failed \\
\hline
\end{tabular}
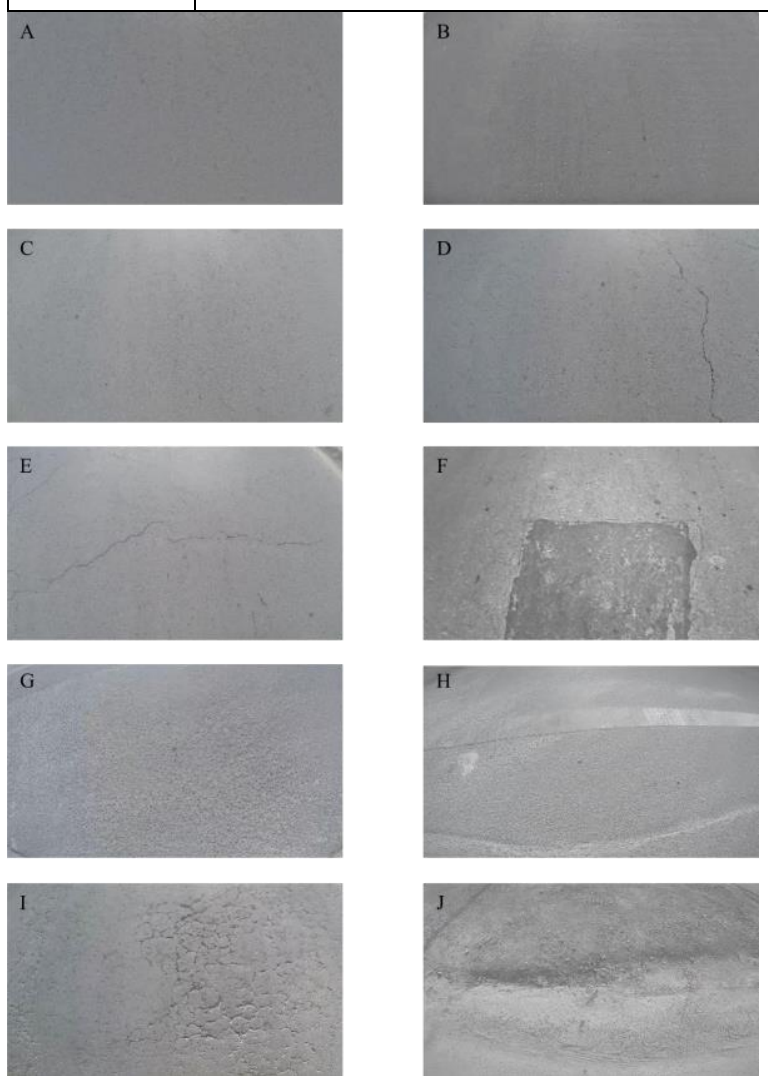

Figure 4: Frames from the examined roadsections

\section{Traffic Volume Index}

A vision-based method for traffic monitoring, provides the data needed for the development of a novel traffic volume index (TVI) (Figure 5). An already developed algorithm, called "Detecting Cars Using Gaussian Mixture Models" (The MathWorks, Inc., 2010), included in the Computer Vision System Toolbox of
MATLAB, formed the basis of the automated traffic monitoring system. The initial algorithm was modified so that it is able to classify vehicles into three classes (Figure 6) and count the number of vehicles belonging in each class.

Firstly, a video is imported, from which frames are extracted, with the first frames being used to initialize the foreground. Moving objects (foreground) are segmented from the background. The algorithm utilizes the morphological "opening" to remove the noise and to fill gaps in the detected objects. Figure 6 shows the initial foreground mask computed by the detector as well as the improved foreground mask (after the application of morphological opening) for a two-wheeled vehicle, a light vehicle, and a heavy vehicle.

The following step finds the bounding box of every connected element corresponding to a moving vehicle, using the Matlab command "vision.BlobAnalysis" that tracks and computes statistics for a set of connected points in a binary image. Once detected, the vehicles are classified into one of three classes based on the number of connected pixels. The minimum and maximum number of connected pixels, for the examined video resolution $(640 \times 360)$, for each class are the following: 4500 and 11000 for two wheeledvehicles; 11000 and 30000 for light vehicles; and more than 30000 for heavy vehicles, respectively. These numbers were arrived at through a search over the range of 3,000 to 40,000 connected pixels in increments of 100 , to identify the best performing district connectivity count based on the performance of the testing set. Finally, the vehicles of each class are counted. This method has an overall recall of $94.07 \%$. 


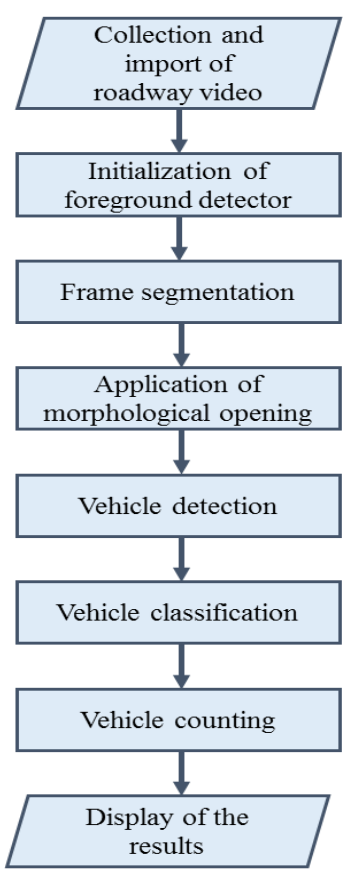

Figure 5: Main stages of the proposed traffic monitoring system
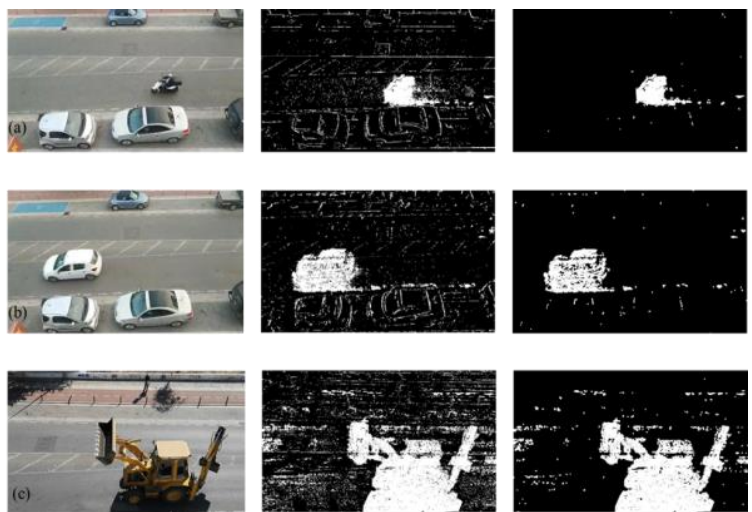

Figure 6: Initial foreground mask and improved foreground mask for: (a) a two-wheeled vehicle; (b) a light vehicle; and (c) a heavy vehicle

Presented herein is the way the counted vehicles are used to estimate the novel TVI. The three different classes of vehicles are transformed into Passenger Car Equivalent (PCE) to estimate traffic flow. Additionally, the daily proportion of $\mathrm{PCE} /$ hour is normalized to a scale from 1-100. This value is combined with the EPCI to form the final maintenance prioritization index (MPI).

PCE is a metric used in Transportation Engineering, to evaluate the traffic flow rate on a roadway. A PCE represents the influence that a mode of transport (vehicle type) has on the traffic variables of headway, speed and density, compared to a single car (lightvehicle) (Ahuja, 2007). Various research studies estimated the PCE of different modes of transport. Table 3 represents the PCE values of the three vehicle classes examined by this paper, as introduced by
(Webster, 1958), Tiwari et al., (2000), Minh and Sano, (2003), Prasetijo (2007) and Partha et al. (2009). The median PCE value of these studies, for each class, is used for the purpose of this study (last row of Table 3 ).

Table 3: PCE values, proposed by various research studies and the current work

\begin{tabular}{|c|c|c|c|}
\hline Study & $\begin{array}{c}\text { Two-wheeled } \\
\text { vehicles }\end{array}$ & $\begin{array}{l}\text { Light } \\
\text { vehicles }\end{array}$ & $\begin{array}{l}\text { Heavy } \\
\text { vehicles }\end{array}$ \\
\hline $\begin{array}{c}\text { Webster } \\
(1958)\end{array}$ & 0.3 & 1 & 2.25 \\
\hline $\begin{array}{c}\text { Tiwari et al. } \\
(2000)\end{array}$ & 0.4 & 1 & 2.4 \\
\hline $\begin{array}{c}\text { Minh and } \\
\text { Sano (2003) }\end{array}$ & 0.29 & 1 & - \\
\hline $\begin{array}{c}\text { Prasetijo } \\
(2007)\end{array}$ & 0.3 & 1 & 3.1 \\
\hline $\begin{array}{c}\text { Saha et al. } \\
(2009)\end{array}$ & - & 1 & 2.16 \\
\hline $\begin{array}{c}\text { Proposed } \\
\text { system }\end{array}$ & 0.3 & 1 & 2.3 \\
\hline \multicolumn{2}{|c|}{} & & \\
\hline
\end{tabular}

After automatically counting all vehicles of each class and calculating the daily proportion of PCE/hour for an examined road lane, a traffic volume index is calculated. Lane capacity varies due to a number of conditions, such as lane width, neighboring lanes, elements next to the road, number of driveways, presence of parking and speed limits. The range of lane capacity is between 1000 and 4800 PCE/hour. Normally lane capacity is between 1500 and 2400 passenger cars per hour (Austroads, 2013).

The aforementioned lane capacity numbers serve as guides for the normalization of the proportion of PCE/hour to a range between 1 and 100 (Equation 2). The maximum number of $4800 \mathrm{PCE} / \mathrm{hour}$ is transformed into the value of 100. Additionally, 2400, 1500 , and $1000 \mathrm{PCE} /$ hour are converted into the traffic volume index values of 90,80 , and 70 , respectively. Everything in between is normalized accordingly. For instance, the values of vehicles/hour between 4800 and 2400 are normalized intro the TVI values between 100 and 90, while the values of vehicles/hour between 2400 and 1500 are normalized intro the TVI values between 90 and 80 .

\section{Maintenance prioritization index}

This paper proposes the evaluation of pavement condition and traffic, dividing a roadway network into lane sections of $50 \mathrm{~m}$. Every section is rated by the proposed EPCI and TVI. The combination of the two indices provides the final MPI, which also ranges from 1 to 100 . The weighted arithmetic mean is utilized for calculating the MPI (Equation 3). 


$$
\mathrm{MPI}=\frac{\mathrm{w} 1 * \mathrm{EPCI}+\mathrm{w} 2 * \mathrm{TVI}}{\mathrm{w} 1+\mathrm{w} 2}
$$

A weighted arithmetic mean is estimated by data points that do not contribute equally to the final average. In case of all weights being equal, then the weighted arithmetic mean equals the arithmetic mean. The current paper proposes an equal contribution of traffic and pavement condition, after advised by pavement experts. However, transportation authorities can utilize the proposed maintenance prioritization method, selecting the weights of EPCI and TVI according to their needs and priorities (pavement condition or traffic volume of a section).

A case study was conducted to test and validate the presented MPI in the roadway network of Nicosia, Cyprus. The EPCI was tested by use of videos acquired from roads of total length of $10 \mathrm{~km}$, with a classification accuracy of $89.2 \%$. However, the TVI was tested in a portion of these roads (of total length of $1 \mathrm{~km}$ ), with an overall recall of $94.07 \%$. Thus, the final MPI, which depends on both EPCI and TVI, was estimated for the common $1 \mathrm{~km}$-length roadways. These roads were divided into 20 equal road sections of 50m-length. Firstly, EPCI, TVI and MPI were calculated for each section, and secondly, sections maintenance was prioritized, with the section with the highest MPI being first in the prioritization list.

\section{Conclusions}

Transportation authorities normally have to select the road sections that they will be maintained amongst many sections, due to budget constraints. This paper proposes a maintenance prioritization technique for 50 m-length sections of roadway lanes. Each section is rated by a novel index (MPI), which is a combination of the proposed pavement condition (EPCI) and traffic volume (TVI) indices. The following characteristics summarize the advantages of the proposed method compared to existing maintenance prioritization systems:

- The EPCI is a continuous variable with a range from 1 to 100.

- The MPI considers not only pavement condition but also traffic volume.

- The maintenance prioritization system provides not only the theory on the calculation of the MPI based on the EPCI and TVI, but also the visionbased tools for automated assessment of pavement condition and traffic.

Future work includes the investigation of other factors that might be taken into account for the prioritization, such as maintenance and rehabilitation cost, and road width. The weights given to each considering factor for calculating the weighted arithmetic mean will also be explored. The final proposed solution should be tested and validated by a greater case study in a real road network.

\section{References}

Ahuja, A.S., 2007. Development of passenger car equivalents for freeway merging sections.

Amarasiri, S., Gunaratne, M., Sarkar, S., 2010. Modeling of crack depths in digital images of concrete pavements using optical reflection properties. J. Transp. Eng. 136, 489-499. https://doi.org/10.1061/(ASCE)TE.19435436.0000095

ASTM International, 2012. ASTM E867-06(2012) Standard Terminology Relating to VehiclePavement Systems | Engineering360 [WWW Document]. URL https://reference.globalspec.com/standard/384855 9/astm-e867-06-2012 (accessed 7.26.18).

Austroads, 2013. Guide to Traffic Management Part 3: Traffic Studies and Analysis.

Bandara, N., Gunaratne, M., 2001. Current and Future Pavement Maintenance Prioritization Based on Rapid Visual Condition Evaluation. J. Transp. Eng. 127 , 116-123. https://doi.org/10.1061/(ASCE)0733947X(2001)127:2(116)

Banharnsakun, A., 2017. Hybrid ABC-ANN for pavement surface distress detection and classification. Int. J. Mach. Learn. Cybern. 8, 699710. https://doi.org/10.1007/s13042-015-0471-1

Bianchini, A., Bandini, P., Smith, D.W., 2010. Interrater reliability of manual pavement distress evaluations. J. Transp. Eng. 136, 165-172. https://doi.org/10.1061/(ASCE)0733947X(2010)136:2(165)

Chen, S.-C., Shyu, M.-L., Zhang, C., 2001. An unsupervised segmentation framework for texture image queries, in: Computer Software and Applications Conference, 2001. COMPSAC 2001. 25th Annual International. IEEE, pp. 569-573.

Christodoulou, S.E., Ellinas, G., MichaelidouKamenou, A., 2009. Minimum moment method for resource leveling using entropy maximization. J. Constr. Eng. Manag. 136, 518-527.

Department of Transport, Tourism and Sport, Government of Ireland, 2013. Rural flexible roads manual department of transport tourism and sport.

Douangphachanh, V., Oneyama, H., 2014. A study on the use of smartphones under realistic settings to estimate road roughness condition. EURASIP J. Wirel. Commun. Netw. 2014, 114. https://doi.org/10.1186/1687-1499-2014-114 
Doycheva, K., Koch, C., König, M., 2016. GPUenabled pavement distress image classification in real time. J. Comput. Civ. Eng. 31, 04016061.

Fwa, T.F., 2005. The handbook of highway engineering. CRC Press.

Gong, H., Cosker, D., 2016. Interactive removal and ground truth for difficult shadow scenes. JOSA A 33, 1798-1811.

Gupte, S., Masoud, O., Martin, R.F., Papanikolopoulos, N.P., 2002. Detection and classification of vehicles. IEEE Trans. Intell. Transp. Syst. 3, 37-47.

Hadjidemetriou, G.M., Christodoulou, S.E., 2019. Vision- and Entropy-Based Detection of Distressed Areas for Integrated Pavement Condition Assessment. J. Comput. Civ. Eng. 33, 04019020. https://doi.org/10.1061/(ASCE)CP.19435487.0000836

Hadjidemetriou, G.M., Christodoulou, S.E., Vela, P.A., 2016. Automated detection of pavement patches utilizing support vector machine classification. IEEE, pp. 1-5. https://doi.org/10.1109/MELCON.2016.7495460

Hadjidemetriou, G.M., Vela, P.A., Christodoulou, S.E., 2018. Automated Pavement Patch Detection and Quantification Using Support Vector Machines. J. Comput. Civ. Eng. 32, 04017073. https://doi.org/10.1061/(ASCE)CP.19435487.0000724

Harris, N.K., González, A., OBrien, E.J., McGetrick, P., 2010. Characterisation of pavement profile heights using accelerometer readings and a combinatorial optimisation technique. J. Sound Vib. 329, 497-508.

Huang, Y., Xu, B., 2006. Automatic inspection of pavement cracking distress. J. Electron. Imaging 15, 013017. https://doi.org/10.1117/1.2177650

Koch, C., Jog, G.M., Brilakis, I., 2012. Automated pothole distress assessment using asphalt pavement video data. J. Comput. Civ. Eng. 27, 370-378.

Kumar, R., Mukherjee, A., Singh, V.P., 2017. Community Sensor Network for Monitoring Road Roughness Using Smartphones. J. Comput. Civ. Eng. $\quad 31$, 04016059. https://doi.org/10.1061/(ASCE)CP.19435487.0000624

Lakušić, S., Brčić, D., Tkalčević Lakušić, V., 2011. Analysis of vehicle vibrations-new approach to rating pavement condition of urban roads. Promet-
TrafficTransportation 23, 485-494.

Li, L., Wang, K.C.P., 2016. Bounding Box-Based Technique for Pavement Crack Classification and Measurement Using $1 \mathrm{~mm}$ 3D Laser Data. J. Comput. Civ. Eng. 30, 04016011. https://doi.org/10.1061/(ASCE)CP.19435487.0000568

Liu, S., Yamada, M., Collier, N. and Sugiyama, M., 2013. Change-point detection in time-series data by relative density-ratio estimation. Neural Networks, 43, pp.72-83.

Lokeshwor, H., Das, L.K., Goel, S., 2014. Robust Method for Automated Segmentation of Frames with/without Distress from Road Surface Video Clips. J. Transp. Eng. 140, 31-41. https://doi.org/10.1061/(ASCE)TE.19435436.0000564

Mathavan, S., Rahman, M., Stonecliffe-Jones, M., Kamal, K., 2014. Pavement raveling detection and measurement from synchronized intensity and range images. Transp. Res. Rec. J. Transp. Res. Board 2457, 3-11. https://doi.org/10.3141/2457-01

Mednis, A., Strazdins, G., Zviedris, R., Kanonirs, G., Selavo, L., 2011. Real time pothole detection using Android smartphones with accelerometers. IEEE, pp. $1-6$. https://doi.org/10.1109/DCOSS.2011.5982206

Minh, C., Sano, K., 2003. Analysis of motorcycle effects to saturation flow rate at signalized intersection in developing countries. J. East. Asia Soc. Transp. Stud. 1211-1222.

Moazami, D., Behbahani, H., Muniandy, R., 2011. Pavement rehabilitation and maintenance prioritization of urban roads using fuzzy logic. Expert Syst. Appl. 38, 12869-12879. https://doi.org/10.1016/j.eswa.2011.04.079

Mohamed, A., Fouad, M.M.M., Elhariri, E., ElBendary, N., Zawbaa, H.M., Tahoun, M., Hassanien, A.E., 2015. RoadMonitor: An intelligent road surface condition monitoring system, in: Intelligent Systems' 2014. Springer, pp. 377-387.

Ndume, V., Mlavi, E., 2017. Multi Criteria Model in Prioritization of Road Maintenance between the Competing Needs - Case Study in Tanzania. IJCSN Int. J. Comput. Sci. Netw. 6.

Ngwangwa, H.M., Heyns, P.S., Breytenbach, H.G.A., Els, P.S., 2014. Reconstruction of road defects and road roughness classification using Artificial Neural Networks simulation and vehicle dynamic 
responses: Application to experimental data. J. Terramechanics 53, 1-18.

Pantha, B.R., Yatabe, R., Bhandary, N.P., 2010. GISbased highway maintenance prioritization model: an integrated approach for highway maintenance in Nepal mountains. J. Transp. Geogr. 18, 426-433. https://doi.org/10.1016/j.jtrangeo.2009.06.016

Partha, S., Mahmud, H., Hossain, Q., Islam, M., 2009. Passenger car equivalent (PCE) of through vehicles at signalized intersections in Dhaka Metropolitan City, Bangladesh. IATSS Res. 33, 99-104.

Prasetijo, J., 2007. Capacity and Traffic Performance of Unsignalized Intersections under Mixed Traffic Conditions. (Ph. D Thesis). Ruhr-University, Bochum.

Radopoulou, S.C., Brilakis, I., 2016. Automated Detection of Multiple Pavement Defects. J. Comput. Civ. Eng. 04016057. https://doi.org/10.1061/(ASCE)CP.19435487.0000623

Reddy, B.B., Veeraragavan, A., 2001. Priority ranking model for managing flexible pavements at network level. J. Indian Roads Congr. 62, 379.

Ryu, S.-K., Kim, T., Kim, Y.-R., 2015. Image-Based Pothole Detection System for ITS Service and Road Management System. Math. Probl. Eng. 2015, 1-10. https://doi.org/10.1155/2015/968361

Seraj, F., van der Zwaag, B.J., Dilo, A., Luarasi, T., Havinga, P., 2014. RoADS: A road pavement monitoring system for anomaly detection using smart phones, in: Big Data Analytics in the Social and Ubiquitous Context. Springer, pp. 128-146.

Shah, Y.U., Jain, S.S., Parida, M., 2014. Evaluation of prioritization methods for effective pavement maintenance of urban roads. Int. J. Pavement Eng. 15 , $238-250$. https://doi.org/10.1080/10298436.2012.657798

Shannon, C.E., 1948. A Mathematical Theory of Communication. Bell Syst. Tech. J. 27, 379-423. https://doi.org/10.1002/j.15387305.1948.tb01338.x

The MathWorks, Inc., 2010. Detecting Cars Using Gaussian Mixture Models.

Tiwari, G., Fazio, J., Pavitravas, S., 2000. Passenger car units for heterogeneous traffic using a modified density method, in: Fourth International Symposium on Highway Capacity. pp. 246-257.
Information Center, University of WisconsinMadison.

Webster, F.V., 1958. A Controlled experiment on the capacity of junctions with traffic signals. Road Res. Lab Res Note No RN3313FVW BR 587.

Xu, Z., Zhao, X., Li, H., Wang, Z., Zhang, M., 2016. Initial Classification Algorithm for Pavement Distress Images Using Features Fusion of Texture and Shape.

Yamada, M., Suzuki, T., Kanamori, T., Hachiya, H. and Sugiyama, M., 2013. Relative density-ratio estimation for robust distribution comparison. Neural computation, 25(5), pp.1324-1370.

Yan, W.Y., Yuan, X.-X., 2017. A low-cost videobased pavement distress screening system for lowvolume roads. J. Intell. Transp. Syst. 1-14.

Ying, L., Salari, E., 2010. Beamlet transform-based technique for pavement crack detection and classification: beamlet transform-based technique. Comput.-Aided Civ. Infrastruct. Eng. 25, 572-580. https://doi.org/10.1111/j.1467-8667.2010.00674.x

Yu, B.X., Yu, X., 2006. Vibration-based system for pavement condition evaluation, in: Applications of Advanced Technology in Transportation. pp. 183189.

Zhang, A., Wang, K.C.P., Fei, Y., Liu, Y., Tao, S., Chen, C., Li, J.Q., Li, B., 2018. Deep LearningBased Fully Automated Pavement Crack Detection on 3D Asphalt Surfaces with an Improved CrackNet. J. Comput. Civ. Eng. 32, 04018041. https://doi.org/10.1061/(ASCE)CP.19435487.0000775

Zhang, A., Wang, K.C.P., Li, B., Yang, E., Dai, X., Peng, Y., Fei, Y., Liu, Y., Li, J.Q., Chen, C., 2017. Automated Pixel-Level Pavement Crack Detection on 3D Asphalt Surfaces Using a Deep-Learning Network. Comput.-Aided Civ. Infrastruct. Eng. 32, 805-819. https://doi.org/10.1111/mice.12297

Zhang, J., Wang, F.-Y., Wang, K., Lin, W.-H., Xu, X., Chen, C., 2011. Data-driven intelligent transportation systems: A survey. IEEE Trans. Intell. Transp. Syst. 12, 1624-1639.

Zhou, J., Huang, P.S., Chiang, F.-P., 2006. Waveletbased pavement distress detection and evaluation. Opt. Eng. 45, 027007-027007.

Walker, D., 2002. PASER manual. Transportation 